\title{
EFECTO DEL RALEO SOBRE EL CRECIMIENTO Y LA DENSIDAD DE LA MADERA DE Pinus taeda IMPLANTADO EN MISIONES, ARGENTINA
}

\author{
EFFECT OF THINNING TREATMENT ON GROWTH AND WOOD DENSITY OF \\ Pinus taeda PLANTED IN MISIONES, ARGENTINA
}

\author{
Rodolfo Martiarena ${ }^{1}$ Ernesto Crechi ${ }^{2}$ Martín Pinazo $^{3}$ Alejandra Von Wallis ${ }^{4}$ Jorge Marquina ${ }^{5}$ \\ Silvia Monteoliva ${ }^{6}$
}

\section{RESUMEN}

El mayor incremento en crecimiento de la plantación puede causar variación en las propiedades físicas de la madera, pudiendo disminuir la calidad de la misma para la industria. El objetivo del presente trabajo es evaluar el efecto de los tratamientos de raleo sobre el crecimiento y densidad de la madera de Pinus

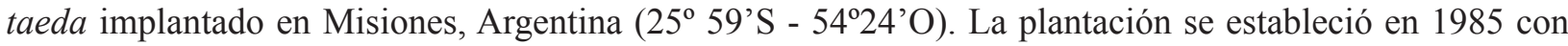
densidad inicial de 1644 pl.ha ${ }^{-1} \mathrm{y}$ se manejó con tres intensidades de raleo $(0,33$ y $66 \%$ del área basal del testigo sin ralear), bajo un diseño de bloques completos al azar ( 3 bloques). A los 20 años se efectuó tala rasa, cuyas densidades eran $711(0 \%), 364(33 \%)$ y 122 (66 \%) plantas por hectárea, momento en el cual se seleccionaron 45 árboles para determinar la densidad básica de la madera. La misma se determinó a través de la realización de 2.700 probetas. El crecimiento de la plantación fue afectado por la intensidad de raleo, registrando diámetros promedio de $28,7,34,8$ y $45,9 \mathrm{~cm}$, respectivamente, en los tratamientos 0 , 33 y $66 \%$ de raleo. El volumen de la plantación, al momento del apeo, fue superior en el tratamiento sin raleo, no obstante el tratamiento $66 \%$ de raleo generó mayor cantidad de madera de grandes dimensiones. La densidad básica como promedio simple y ponderado por volumen no presentó diferencias significativas entre tratamientos. Los valores de densidad ponderada fueron de 0,406, 0,418 y 0,420 g. $\mathrm{cm}^{-3}$ para 1 los tratamientos 0,33 y $66 \%$ de raleo, respectivamente, mientras que el análisis de correlación entre diámetro y densidad no fue significativo. El estudio mostró que la intensidad de raleo puede ser manejada para maximizar la producción, manteniendo idéntica la calidad de la madera para la industria.

Palabras clave: plantaciones; tratamientos silvícolas; propiedades de la madera; pino.

\section{ABSTRACT}

The greatest increase in growth of the plantation may change the physical properties of wood, and therefore may decrease the wood quality for industry. The current paper aimed to evaluate the effect of thinning

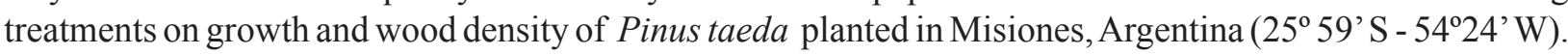
The plantation was established in 1985 with 1644 plants per hectare and three thinning intensity treatments were used $(0 \%, 33 \%$ and $66 \%$ of the remaining basal area), under design randomized complete blocks ( 3

1 Ingeniero Forestal, Msc., Instituto Nacional de Tecnología Agropecuaria (INTA), Av. El Libertador 2472, 3384, Montecarlo, Misiones, Argentina. ramartiarena@montecarlo.inta.gov.ar

2 Ingeniero Forestal, Msc., Instituto Nacional de Tecnología Agropecuaria (INTA), Av. El Libertador 2472, 3384, Montecarlo, Misiones, Argentina. ecrechi@montecarlo.inta.gov.ar

3 Ingeniero Forestal, Msc.,Instituto Nacional de Tecnología Agropecuaria (INTA), Av. El Libertador 2472, 3384, Montecarlo, Misiones, Argentina. mpinazo@montecarlo.inta.gov.ar

4 Ingeniero Forestal, Instituto Nacional de Tecnología Agropecuaria (INTA), Av. El Libertador 2472, 3384, Montecarlo, Misiones, Argentina. avonwallis@montecarlo.inta.gov.ar

5 Ingeniero Forestal, Universidad Nacional de La Plata, Facultad de Ciencias Agrarias y Forestales, CC 31 (1900), La Plata, Provincia de Buenos Aires, Argentina. jmarqui@agro.unlp.edu.ar

6 Licenciada en Biología, Dr ${ }^{\mathrm{a}}$., Facultad de Ciencias Agrarias y Forestales, Universidad Nacional de La Plata, CC 31 (1900), La Plata, Provincia de Buenos Aires, Argentina. Consejo Nacional de Investigaciones Científicas y técnicas (CONICET). smonteoliva@yahoo.com.ar 
blocks). The harvest was performed at rotation age (20 years-old), on parcels with 711(0\%), $364(33 \%)$ and $122(66 \%)$ plants per hectare. Forty-five trees were selected to determine the basic wood density (15 trees per treatments, 2700 samples). Thinning significantly affected the growth of the plantation, with average diameters of $45.9 \mathrm{~cm}, 34.8 \mathrm{~cm}$ and $28.7 \mathrm{~cm}$ in $0 \%, 33 \%$ and $66 \%$ thinning treatments, respectively. The volume of the plantation was higher in the unthinned treatment, while $66 \%$ thinning produced a deficiently place occupation, generating greater amounts of large wood. For simple and weighted wood density the differences between thinning treatments were not significant. The weighted density values were 0.406 , 0.418 and $0.420 \mathrm{~g} . c m^{-3}$ for $0 \%, 33 \%$ and $66 \%$ thinning treatments, respectively. The correlation analysis carried out between the diameter and density was not significant. The intensity of thinning can be managed to maximize the production without any modification in the wood quality for industry.

Keywords: plantations; silvicultural treatments; wood properties; pine.

\section{INTRODUCCIÓN}

El género Pinus es el más cultivado en Argentina, superando las 600.000 ha entre las provincias de Misiones y Corrientes, concentrado mayoritariamente desde los $29^{\circ} \mathrm{S}$ en la provincia de Corrientes, hasta los $25^{\circ} 30^{\prime} \mathrm{S}$ en la provincia de Misiones. La especie con mayor superficie cultivada en la provincia de Misiones es el Pinus taeda L., abarcando 306.000 ha (SIFIP, 2010), correspondientes aproximadamente al $11 \%$ de la superficie total de la provincia, mientras que Corrientes totaliza 263.000 ha forestadas con el género Pinus (CFI, 2009).

El ciclo de rotación de Pinus taeda en Argentina, oscila los 20 años, período en el cuál se aplican 3 o 4 raleos. Si bien el raleo tiene como objetivo principal estimular el crecimiento de los árboles que quedan y aumentar la producción de madera de calidad (NUTTO et al., 2006; BLANCO et al., 2006), la producción de biomasa alcanza su máximo con la mayor ocupación del sitio, es decir, sin raleos (PINAZO et al., 2008).

Las plantaciones cuyo manejo incorpora al raleo, producen mayor espaciamiento para el crecimiento individual de las plantas, lo cual afecta el tamaño, forma y estructura del fuste principal. El fuste resulta más cónico y con grandes ramas que permanecen vivas en la parte inferior del tronco (MOORE et al., 2009), pudiendo también afectar la estructura de la madera producida, alterando su potencial uso final por parte de las industrias forestales (JYSKE et al., 2010).

La densidad de la madera es la propiedad más importante al definir la calidad del leño, ya que de ella dependen otras propiedades como las resistencias mecánicas y los rendimientos en pulpa (BARNETT y JERONIMIDIS, 2003). El raleo puede afectar la densidad de la madera, pero dicha relación es extremadamente compleja, porque en ella intervienen aspectos genéticos y del ambiente (TODARO y MACHIONI, 2011).

Los antecedente bibliográficos, a nivel internacional, muestran resultados contradictorios del efecto de los tratamientos de raleo sobre la densidad de la madera en diferentes especies de coníferas, ya que puede disminuir la densidad de la madera con mayor intensidad de raleo (CAO et al., 2008; JYSKE et al., 2010; TODARO y MACHIONI, 2011), puede aumentar (MACPEAK et al., 1990; WODZICKI, 2001) o no mostrar relación entre ambas variables (JAAKKOLA et al., 2006;GULLER, 2007; MOORE et al., 2009; LASSERRE et al., 2009). En Argentina, y para esta especie no hay estudios que determinen estas relaciones.

La hipótesis de trabajo es que el mayor crecimiento de la plantación generado por la aplicación de raleos intensos y en repetidas oportunidades a lo largo del turno de rotación, disminuye los valores de densidad básica de la madera. Para dar respuesta a la hipótesis planteada, el objetivo es determinar el efecto de los tratamientos de raleo sobre el crecimiento y su relación con la densidad básica de la madera de Pinus taeda de 20 años de edad, cultivado en Misiones, Argentina.

\section{MATERIALES Y MÉTODOS}

\section{Caracterización del sitio}

El trabajo se realizó en un predio de la Empresa LIPSIA S.A. denominado Campo Elena, cercano a la localidad de Wanda, Departamento

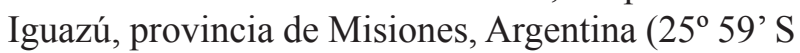
y $54^{\circ} 24^{\prime} \mathrm{O}$ ). 
El suelo se clasifica como Kandiudult (SOIL SURVEY STAFF, 2006) y se caracteriza por un desarrollo en profundidad mayor a los dos metros, con una secuencia de horizontes A-Bt-C, de color rojo, libre de pedregosidad y fragmentos gruesos, permeabilidad moderada y bien drenado. El clima de la región se caracteriza por una temperatura media anual de $21^{\circ} \mathrm{C}$ y precipitaciones que varían alrededor de $2.000 \mathrm{~mm}$ anuales, con régimen isohigro (INTA, 1990).

\section{Caracterización del rodal}

La plantación se realizó en el año 1985 con Pinus taeda de origen Marion (EE.UU.) con densidad inicial de 1.644 plantas por hectárea $\left(\right.$ pl.ha $\left.^{-1}\right)$. En 1991 se efectuó un raleo bajando la densidad del rodal a 894 pl.ha $^{-1}$. Bajo un diseño experimental de bloques completos al azar con tres repeticiones, se instaló en el año 1993 un ensayo de intensidad de raleo, aplicándose tres tratamientos: tratamiento testigo con $0 \%$ de raleo (T1), tratamiento intermedio con 33 $\%$ de intensidad de raleo (T2) y, tratamiento fuerte con $66 \%$ de intensidad de raleo (T3), tomando de referencia el área basal del testigo. Los tratamientos se aplicaron cada cuatro años, efectuándose a los 8 años (1993), 12 años (1997) y 16 años (2001) años de edad. El tamaño neto de cada una de las nueve parcelas evaluadas fue de $806 \mathrm{~m}^{2}$, efectuándose el raleo en todas las ocasiones por lo bajo. La cosecha (tala rasa) se efectuó en el año 2005 con 711 pl.ha $^{-1}$, 364 pl.ha $^{-1}$ y 122 pl.ha $^{-1}$ en los tratamientos $0 \%, 33 \%$ y $66 \%$ de raleo, respectivamente, con un diámetro promedio a la altura del pecho (DAP) que alcanzó los $28,7 \mathrm{~cm} ; 34,6 \mathrm{~cm}$ y $45,9 \mathrm{~cm}$ para el mismo orden de tratamientos, con tasas de crecimiento de los árboles en pie de $32,9 \mathrm{~m}^{3}$.ha año ${ }^{-1} ; 24,2 \mathrm{~m}^{3}$.ha año-1 $\mathrm{y}$ $13,8 \mathrm{~m}^{3}$.ha año ${ }^{-1}$.

\section{Selección de los árboles}

Se apearon 45 árboles, correspondiendo 15 a cada tratamiento de raleo y, a su vez, cinco a cada una de las tres repeticiones de cada tratamiento. En cada una de las repeticiones los árboles a ser apeados se seleccionaron de acuerdo a la distribución diamétrica, obtenida con mediciones previas de la totalidad de los individuos de cada repetición. Para ello, se definieron tres clases diamétricas en cada repetición y se apeó un árbol en la clase diamétrica inferior, un árbol en la clase diamétrica superior y tres árboles en la clase diamétrica intermedia de mayor frecuencia arbórea, seleccionados de forma al azar dentro de cada clase diamétrica.

\section{Determinación de la densidad básica de la madera}

Cada árbol fue cortado a $10 \mathrm{~cm}$ sobre el suelo y su fuste trozado en secciones a longitudes variables, según utilidad en la industria de la empresa propietaria, hasta los $5 \mathrm{~cm}$ de diámetro. Los límites inferiores de corte de cada una de las trozas correspondieron respectivamente desde la base a 0,1 m (troza 1), 3,5 m (troza 2), 5,5 m (troza 3), 8,5

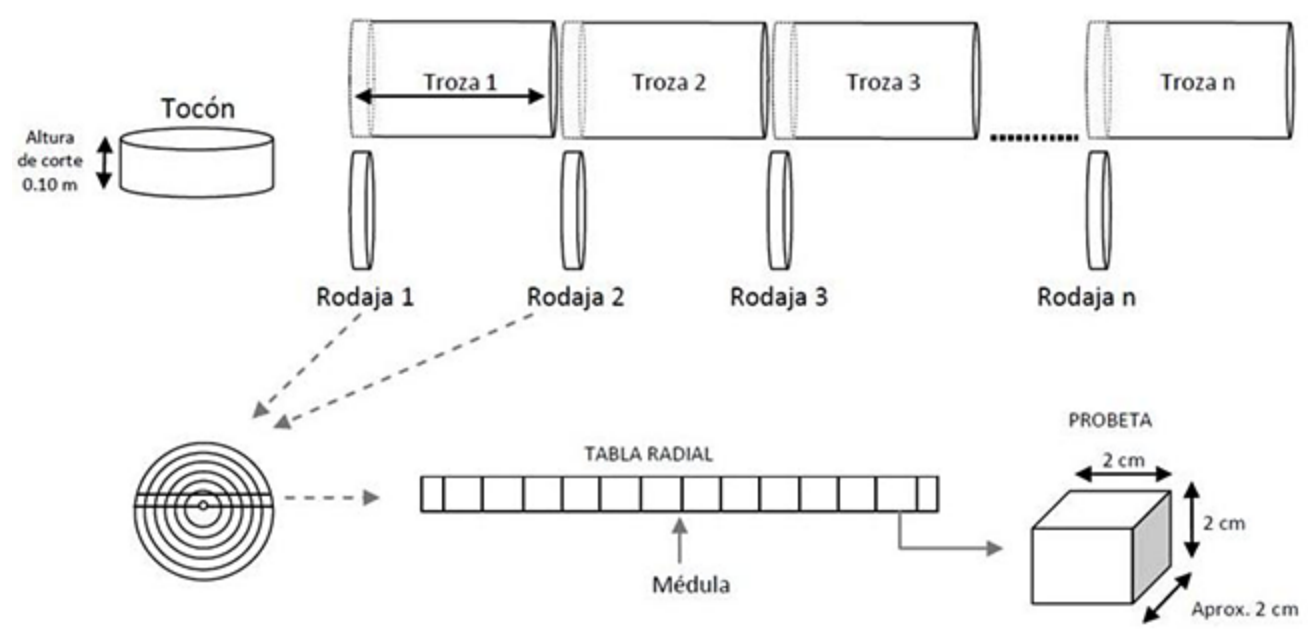

FIGURA 1: Esquema de trozado de los árboles y obtención de las probetas para determinar la densidad básica de la madera.

FIGURE 1: Diagram of trees sampling and the obtaining of specimens to determine the basic wood density. 
$\mathrm{m}$ (troza 4), 12,5 m (troza 5), 16,5 m (troza 6), $21 \mathrm{~m}$ (troza 7) y, mayor a $21 \mathrm{~m}$ hasta los $5 \mathrm{~cm}$ de diámetro en el fuste. En cada corte se extrajo una rodaja del límite inferior de la troza, para la determinación de la densidad básica de la madera a lo largo del fuste. De cada una de estas rodajas, se sacó una tabla radial de $2 \mathrm{~cm}$ de ancho, atravesando la totalidad de los anillos de crecimiento a ambos lados de la médula. Sobre esta tabla y a partir del centro medular, se cortaron hacia ambos lados probetas de $2 \mathrm{~cm}$ de largo por $2 \mathrm{~cm}$ de ancho y espesor variable con aproximación a los $2 \mathrm{~cm}$. (Figura 1).

Se obtuvieron 2.700 probetas en total y cada una de ellas se sumergió en agua durante siete días para lograr el volumen saturado, el cual fue determinado por inmersión en agua, aplicando el principio de Arquímedes. Luego se sometieron a estufa a $103 \pm 2{ }^{\circ} \mathrm{C}$ hasta peso constante, para obtener luego el peso seco de las mismas. Con ambos valores, peso seco (Po) y volumen saturado (Vs), se determinó la densidad básica de la madera (Db) como la relación Po / Vs.

Luego de obtenido el valor de densidad básica de la madera para cada probeta, se calculó la densidad básica del árbol completo como un promedio aritmético simple y como un promedio ponderado (fórmulas [1], [2] y [3])

$$
\begin{aligned}
& D h=\frac{\sum_{p=1}^{n} \frac{P_{o p}}{V s p}}{n} \\
& D s=\frac{\sum_{h=1}^{n} D h}{n}
\end{aligned}
$$

Donde: $\mathrm{Dh}=$ densidad básica a la altura de muestreo $h$; Po $p=$ peso seco de la probeta a la altura $\mathrm{h} ; \mathrm{Vs} \mathrm{p}=$ volumen saturado de la probeta a la altura $\mathrm{h} ; \mathrm{N}=$ número de probetas radiales para la altura $\mathrm{h}$; Ds $=$ densidad básica simple del árbol completo.

Los valores medios ponderados de densidad de la madera para el árbol completo (densidad ponderada) son el resultado de la ponderación de la densidad de sus secciones transversales (rodajas) a diferentes alturas por los volúmenes de la sección cónica respectiva según la siguiente fórmula:

$$
D w=\frac{\sum_{h=1}^{8} D h \cdot V t}{\sum_{h=1}^{8} V t}
$$

Donde: $\mathrm{Dw}=$ densidad básica ponderada del árbol completo; $\mathrm{Dh}=$ densidad básica a la altura de muestreo $\mathrm{h} ; \mathrm{Vt}=$ volumen de la troza que contiene la altura de muestreo $\mathrm{h}$.

En cada uno de los tratamientos de raleo se calculó la densidad básica de la madera. La misma se determinó como el promedio aritmético simple de las diferentes alturas muestreadas, mientras que la densidad ponderada se efectuó teniendo en cuenta el volumen del árbol.

Los datos se analizaron bajo el análisis de variancia y las medias se compararon con la prueba de Tukey con un nivel de significancia de 0,05. Se realizaron correlaciones de Pearson entre los diámetros y las densidades.

\section{RESULTADOS}

La Figura 2 muestra los registros de la evolución de las variables dasométricas del rodal en diferentes tratamientos de intensidad de raleo para los períodos de las intervenciones $(8,12,16$ y 20 años de edad). El número de plantas en el rodal $(\mathrm{N})$ decreció con el aumento de la intensidad de raleo (Figura 2A), con diferencias significativas $(P<0,001)$ al momento de realizado el estudio (20 años de edad), mientras que el DAP promedio en el mismo momento aumentó significativamente $(P<$ $0,001)$, también con el aumento de la intensidad de raleo (Figura 2B). En la Figura 2C se observa que el área basal $(\mathrm{G})$ del tratamiento sin raleo (T1) se estabilizó en el período 16 a 20 años, mientras que los tratamientos con raleo (T2 y T3) mantuvieron la tendencia creciente. No obstante las diferencias en valores absolutos en el período mencionado, al momento del estudio, las diferencias fueron significativamente diferentes entre tratamientos $(P$ $<0,001)$. La Figura $2 \mathrm{E}$ muestra que el $\mathrm{G}$ acumulada ( $\mathrm{G}$ existente sumado a lo extraído durante los raleos) hasta los 16 años de edad fue superior en el tratamiento sin raleo, mostrando valores inferiores respecto de los demás tratamientos a partir de ese momento. El volumen (V) fue superior para los tres tratamientos en cada una de las intervenciones, respecto del momento de intervención anterior (Figura 2D), siendo significativamente mayor el V registrado en los tratamientos en que se aplicó raleo $(P<0,001)$, manteniendo la misma tendencia el $\mathrm{V}$ acumulado (V existente más lo extraído durante los raleos) a los 20 años de edad (Figura 2F). La diferencia existente en el cálculo del volumen 
presente y volumen acumulado del tratamiento sin aplicación de raleo (T1), de aproximadamente 48 $\mathrm{m}^{3}$, se debe a la extracción realizada en forma de raleo, previo a la instalación del ensayo (Figuras 2 D y 2 F).

El análisis de los 45 árboles apeados para el estudio de densidad mostró que el crecimiento diamétrico en la base del árbol, manifestó diferencias significativas entre tratamientos $(P<0,001)$. El volumen del árbol individual de los ejemplares apeados, también manifestó diferencias significativas $(P<0,001)$ entre tratamientos (Tabla 1).

La densidad simple y densidad ponderada,
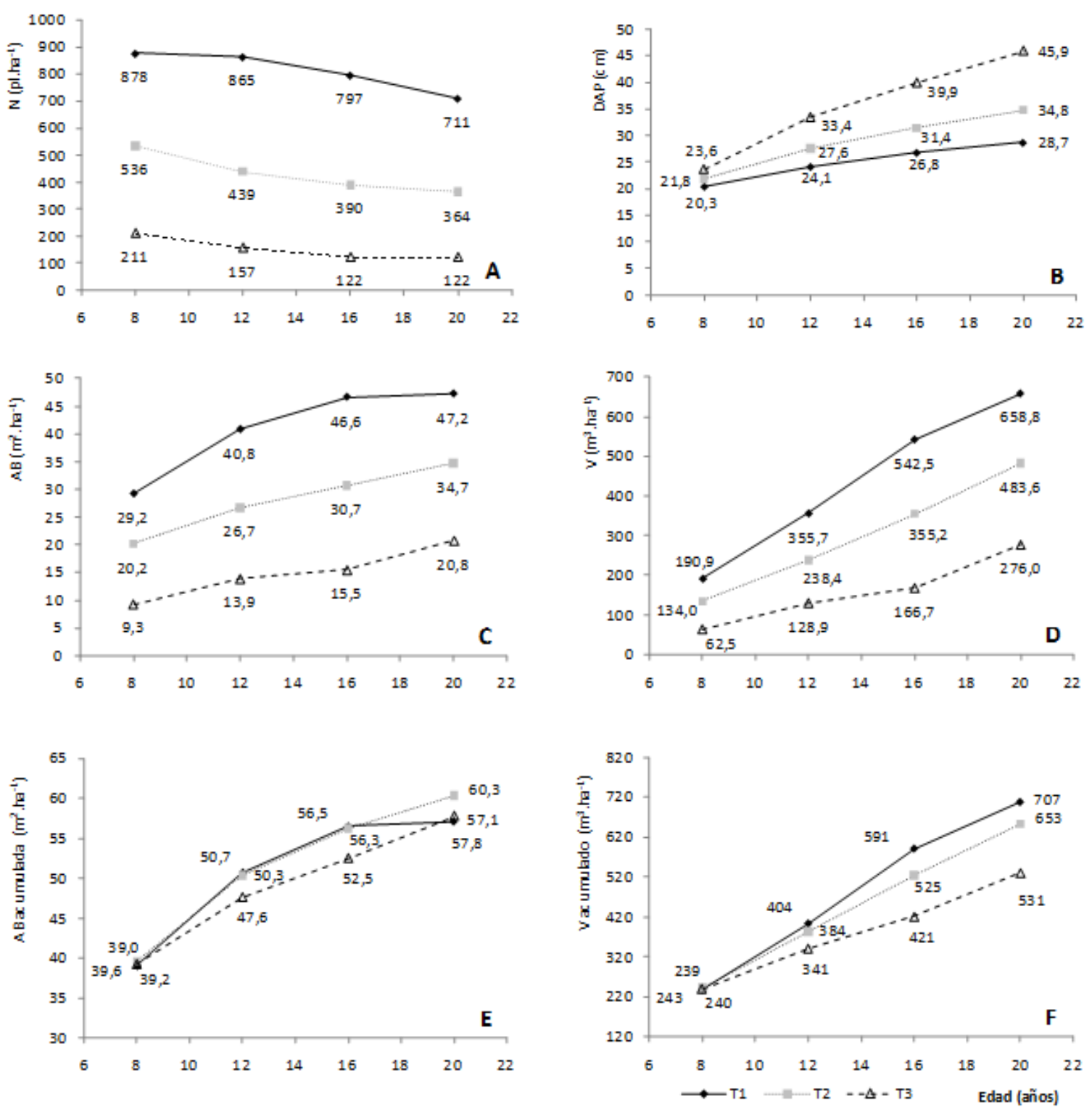

FIGURA 2: Variación de los parámetros dasométricos en función de la intensidad de raleo de Pinus taeda entre los 8 y 20 años de edad. A) Número de plantas por hectárea. B) Diámetro promedio a la altura de pecho. C) Área basal existente. D) Volumen existente. E) Área basal acumulada (área basal existente sumado a lo extraído durante los raleos). F) Volumen acumulado (volumen existente más lo extraído durante los raleos).

FIGURE 2: Dasometric parameters variation as a function of thinning intensity of 8 to 20 years-old Pinustaeda. A) Number of plants per hectare. B) Average diameter at breast high. C) Existing basal area. D) Existing volume. E) Accumulated basal area (remainder BA + thinned BA).F) Accumulated V (remainder V + thinned V). 
TABLA 1: Valores promedio de diámetro basal sin corteza, volumen de fuste del árbol individual sin corteza, densidad básica simple y densidad básica ponderada en función del tratamiento de raleo aplicado durante el turno de rotación de Pinus taeda.

TABLE 1: Average values of basal diameter without bark (BD); stem volume of tree without bark (V) and simple basic density (SD) and weighted basic density (WD) per thinning treatment.

\begin{tabular}{ccccc}
\hline Tratamiento & $\begin{array}{c}\text { Diámetro basal } \\
\text { sin corteza }(\mathrm{cm})\end{array}$ & $\begin{array}{c}\text { Volumen sin } \\
\text { corteza }\left(\mathrm{m}^{3}\right)\end{array}$ & $\begin{array}{c}\text { Densidad básica } \\
\text { simple }\left(\mathrm{g} \cdot \mathrm{cm}^{-3}\right)\end{array}$ & $\begin{array}{c}\text { Densidad básica } \\
\text { ponderada }\left(\mathrm{g} \cdot \mathrm{cm}^{-3}\right)\end{array}$ \\
\hline $0 \%$ raleo & $36,67(10,33) \mathrm{a}$ & $0,80(0,54) \mathrm{a}$ & $0,41(0,02) \mathrm{a}$ & $0,41(0,03) \mathrm{a}$ \\
$33 \%$ raleo & $42,87(9,68) \mathrm{a}$ & $1,16(0,37) \mathrm{a}$ & $0,42(0,02) \mathrm{a}$ & $0,42(0,03) \mathrm{a}$ \\
$66 \%$ raleo & $56,07(8,36) \mathrm{b}$ & $1,65(0,42) \mathrm{b}$ & $0,42(0,04) \mathrm{a}$ & $0,42(0,03) \mathrm{a}$
\end{tabular}

Em que: Letras diferentes presentan diferencias significativas $(P<0,05)$ entre tratamientos, según prueba de Tukey. Entre paréntesis desvíos estándar.

no mostraron diferencias significativas entre tratamientos $(P=0,408$ y $P=0,364$, respectivamente), cuyos valores aumentaron con el aumento de la intensidad de raleo (Tabla 1).

En el análisis conjunto de la totalidad de árboles y en todas sus alturas de muestreo (Figura $3 \mathrm{~A}$ ), no se halló correlación entre diámetro y densidad básica de la madera $(\mathrm{r}=0,008 ; P=$ 0,892). Sin embargo, se observó, que el análisis de correlación realizado entre el diámetro del árbol a la base $(0,1 \mathrm{~m})$ y su respectiva densidad (Figura
3B) mostró una tendencia a disminuir la densidad de la madera con el incremento del crecimiento $(\mathrm{r}=-0,40 ; P=0,006)$.

La densidad básica de la madera a lo largo del fuste se mostró estadísticamente similar en función del tratamiento de raleo aplicado. El valor promedio de los tres tratamientos en la troza 1 fue de $0,46(0,07) \mathrm{g} \cdot \mathrm{cm}^{-3}$, en las cinco trozas centrales alcanzó $0,41(0,01) \mathrm{g} . \mathrm{cm}^{-3}$, mientras que en la troza 7 el valor fue de $0,44(0,02)$ g.cm $\mathrm{cm}^{-3}$.
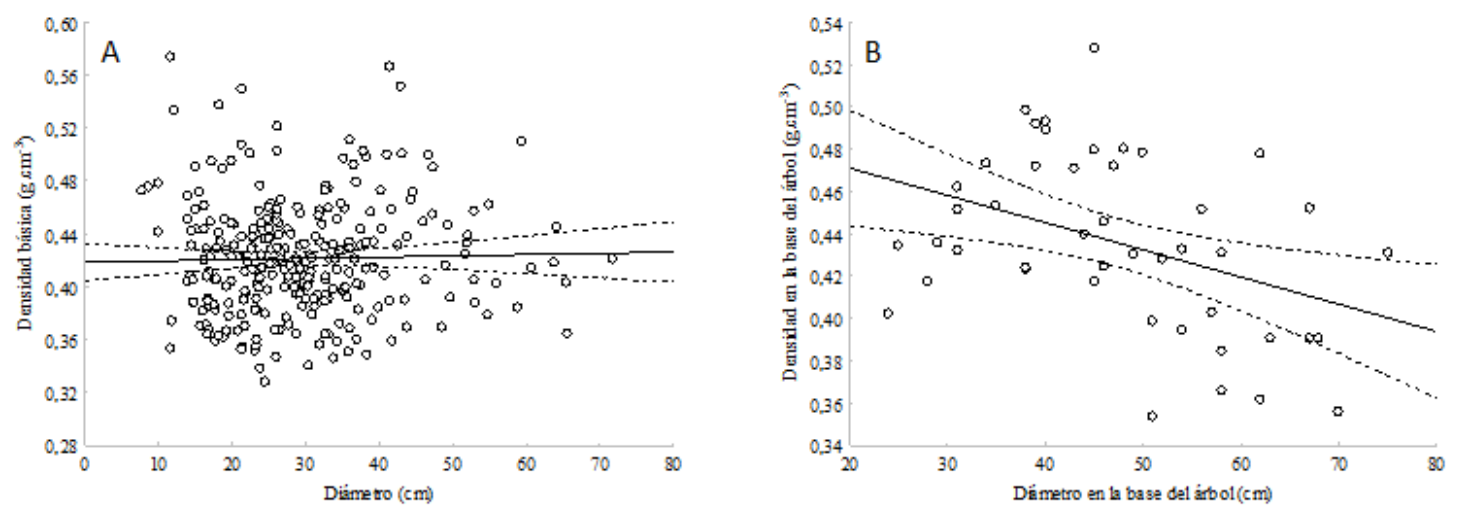

FIGURA 3: Densidad básica de la madera en función del diámetro del fuste. A) Relación entre diámetro de la sección de muestreo y su respectiva densidad básica de la madera a lo largo del fuste $(\alpha=95 \% ; \mathrm{n}=279)$. B) Relación entre diámetro a la base del árbol y su respectiva densidad básica dela madera $(\alpha=95 \% ; n=45)$.

FIGURE 3: Basic wood density as a function of stem diameter. A) Relationship between diameter of sample section and its basic wood density along the stem $(\alpha=95 \% ; n=279)$. B) Relationship between tree base diameter and its basic wood density $(\alpha=95 \% ; n=45)$. 


\section{DISCUSIÓN}

La aplicación sucesiva de raleos durante el ciclo de rotación de Pinus taeda permite aumentar el diámetro promedio en las parcelas tratadas. El tratamiento de raleo intenso (T3) registra un incremento diamétrico del $37 \%$ respecto del tratamiento sin raleo (T1), lo cual podría asociarse a un aumento en la producción de madera de calidad (JYSKE et al., 2010). El incremento diamétrico en los tratamientos con aplicación de raleo se debe, principalmente, a la disminución del $81 \%$ del número de plantas, lo cual permite la mejor utilización de los recursos disponibles por los árboles remanentes a nivel de árbol individual (BURKES et al., 2003; MARTIARENA et al., 2011), lo cual también indujo al fortalecimiento de los mismos (SWORD et al., 2004). Como se observa en la Figura 2A, la mortalidad natural en el tratamiento sin raleo entre los 8 y 20 años de edad produjo un descenso en la densidad del rodal de 878 a 711 pl.ha $^{-1}$, cuya mortalidad podría estar relacionado con la competencia por los recursos disponibles (luz, agua y nutrientes), situación que permite inducir que los árboles de las parcelas manejadas con tratamientos de raleo dispusieron de mayor cantidad de recursos.

El volumen presente en cada tratamiento a los 20 años de edad es superior en el tratamiento sin raleo (T1), consecuencia, como ya se explicó, de la ocupación total del sitio en el tratamiento sin raleo, coincidente con los resultados encontrados en otras investigaciones (REY et al.,2001; MERINO et al.,2003; BALBOA et al.,2004; CRECHI et al., 2005). El área basal acumulada en el tratamiento testigo es inferior a los tratamientos con raleos a los 20 años, mientras que el volumen acumulado no se manifiesta de la misma manera. Se asume que este efecto puede deberse a la diferencia en las condiciones individuales de crecimiento de los árboles en cada tratamiento, ya que Schneider et al. (1991), hacen referencia a otros trabajos donde se constata que plantaciones manejadas con raleos fuertes producen menor volumen que plantaciones manejadas con raleos suaves, aun cuando se registran mayores crecimientos en área basal en los tratamientos con raleos fuertes. Esto es debido a que los árboles bajo regímenes intensivos de raleo tienen menor altura y mayor conicidad. En el presente trabajo la altura media de los árboles es 1,4 $\mathrm{m}$ superior en $\mathrm{T} 1$ respecto de $\mathrm{T} 3$ (datos no presentados), mientras que la conicidad no fue evaluada.

La densidad de la madera no es afectada por el aumento de la intensidad de raleo (Tabla 1). Esto indica que se puede obtener el máximo rendimiento del rodal sin afectar la densidad de la madera, principal propiedad al definir la calidad del leño, ya que de ella dependen propiedades como las resistencias mecánicas y los rendimientos en pulpa (BARNETT y JERONIMIDIS, 2003). Guller (2007), reporta que no hay una relación clara entre el incremento en el tamaño de los anillos de crecimiento y la cantidad del leño producido cada año para árboles de Pinus brutia de 33-35 años manejados con dos intensidades de raleo. El estudio de este autor, indica que los árboles de plantaciones con raleo incrementan más del $70 \%$ el diámetro a la altura de pecho y su área basal incrementa más del $20 \%$, sin mostrar diferencias en la densidad básica de la madera, coincidente con el presente trabajo. También JAAKKOLA et al. (2006) efectuaron un trabajo en Finlandia para determinar la relación diámetro del árbol con la densidad básica de la madera, influenciado por los tratamientos de raleo en Picea abies L. y aplican dos intensidades de raleo removiendo 8 y $29 \%$ del G, quienes pudieron demostrar que la densidad básica de la madera no es significativamente diferente en las diferentes clases diamétricas generadas por el efecto del raleo. Si bien en especies y condiciones ambientales diferentes, los resultados que aquí se presentan coinciden con la mención anterior, ya que independientemente del tratamiento de raleo aplicado, es posible lograr un incremento diamétrico en la base del fuste del árbol de $53 \%$ en el tratamiento con 66 $\%$ de raleo, manteniendo la densidad básica de la madera inalterada. Este resultado deja de manifiesto que la principal troza utilizable por la industria mantiene las propiedades intrínsecas de la especie, independientemente del tratamiento de raleo que se aplique, maximizando la producción de madera de mayor diámetro. Otras investigaciones (MOORE et al., 2009; LASSERRE et al., 2009) también muestran que no existe relación entre el incremento diamétrico y la densidad básica de la madera.

La cantidad de carbohidratos producido por un árbol depende fundamentalmente de la superficie foliar y la habilidad de las raíces de suministrar los nutrientes a las hojas. La relación espacio/superficie de crecimiento por árbol se incrementa después de cada tratamiento de raleo, disminuyendo así la competencia intraespecífica. La bibliografía reporta los más diversos resultados en cuanto a relación 
densidad arbórea del rodal y densidad básica de la madera, indicando que la densidad básica de la madera puede variar como consecuencia del raleo (TODARO y MACCHIONI, 2011).

Algunos autores expresan la relación densidad básica de la madera con el ancho de los anillos de crecimiento (GULLER, 2007; JYASKE et al., 2010; TODARO y MACCHIONI, 2011), incremento en área basal (JAAKKOLA et al., 2006) o volumen (CAO et al., 2008). Mayoritariamente la bibliografía reporta, para coníferas, que el aumento en la intensidad de raleo genera menor densidad básica de la madera (CAO et al., 2008; JYSKE et al., 2010; TODARO y MACCHIONI, 2011), mientras que otros trabajos muestran la tendencia inversa (MACPEAK et al., 1990; WODZICKI, 2001).

\section{CONCLUSIONES}

El crecimiento del Pinus taeda en Misiones, Argentina, responde positivamente ante la aplicación de diferentes intensidades de raleo a lo largo del turno de rotación hasta los 20 años de edad. Ante el significativo incremento de las variables de crecimiento se puede observar que la densidad básica de la madera no varía, por lo que se rechaza la hipótesis de trabajo. Bajo las condiciones de estudio el trabajo muestra que la intensidad de raleo puede ser manejada para maximizar producción, manteniendo idéntica calidad de madera para la industria.

\section{AGRADECIMIENTOS}

A la empresa LIPSIA S.A. por proveer el sitio de trabajo y operarios para el mismo. Al señor Otto Knebel por colaborar en diferentes etapas del trabajo.

\section{REFERENCIAS BIBLIOGRÁFICAS}

BALBOA, M. et al.2004. Temporal variations and distribution of carbon stocks in above-ground tree biomass of Pinusradiataand Pinuspinasterpure stands under different silvicultural alternatives. International IUFRO. Conference on Sustainable Harvest Scenarios in Forest Management. Tale, the Low Tatras, Slovakia, 25-27 Agosto. 2004.

BARNETT, J.; JERONIMIDIS, G. 2003. Wood quality and its biological basis. Oxford, UK. Blackwell Publishing Ltd. 226 p.

BLANCO, J.; BOSCO IMBERT, J.; CASTILLO,
F. 2006. Effects of thinning on nutrient content pools in two Pinussylvestris forests in the western Pyrenees. Scandinavian Journal Forest Research, 21(2): 143-150.

BURKES, E.; WILL, R.; BARRON, G.; TESKEY, R.; BARRY, S. 2003. Biomass partitioning and growth efficiency of intensively managed Pinustaeda and Pinuselliottii stand of different planting densities. Ann. Forest Scientist, 47(2): 224-234.

CAO, T. et al. 2008. Effects of thinning and fertilization on wood properties and economic returns for Norway spruce. Forest Ecology and Management, 256:1280-1289.

CFI. 2009. Primer Inventario Forestal Provincia de Corrientes. Metodología, trabajo de campo y resultados. Consultado: http://www.corrientes.gov. ar/portal/files/Informe\%20Final.pdf

CRECHI, E. et al.2005. Efectos del raleo en Pinustaeda. IDIA XXI. Año V- $\mathrm{N}^{\circ}$ 8: 43-47.

GULLER, B. 2007. The effects of thinning treatments on density, MOE, MOR and maximum crushing strength of PinusbrutiaTen. wood. Ann. Forest Scientist, 64:467-475.

INTA. 1990. Atlas de suelos de la República Argentina.Provincia de Misiones, 2: 111-154.

JAAKKOLA, T.; MAKINEN, H.; SARANPAA, P. 2006. Wood density in Norway spruce: response to timing and intensity of first commercial thinning and fertilization. Forest Ecology and Management, 237:513-521.

JYSKE, T. et al. 2010. Effects of timing and intensity of thinning on wood structure and chemistry in Norway spruce. Holzforschung, 64:81-91.

LASSERRE, J. et al. 2009. Influence of initial planting spacing and genotype on microfibril angle, wood density, fibre properties and modulus of elasticity in Pinusradiata D. Don corewood. Forest Ecology and Management, 237: 1924-1931.

MACPEAK, M.; BURKART, L.; WELDON, D. 1990. Comparison of grade, yield, and mechanical properties of lumber produced from young fastgrown and older slow-grown planted Slash pine.

Forest Product Journal, 40: 11-14.

MARTIARENA, R. et al. 2011. Effectof thinning and harvest type on storage and losses of Phosphorous in Pinustaeda L. plantations in Subtropical Argentina. International Journal of Forestry Research. v. 2011, Art. ID 761532, 10 P. DOI:10.1155/2011/761532.

MERINO, A. et al. 2003. Biomasa arbórea y acumulación de nutrientes en plantaciones de 
$P$. radiata en Galicia. Investigación Agraria.: Sistemas y Recursos Forestales, 12(2): 85-98.

MOORE, J. et al. 2009. Effects of early re-spacing on the physical and mechanical properties of Stika spruce structural timber. Forest Ecology and Management, 258:1174-1180.

NUTTO, L.; SPATHELF, P.; SELING, I. 2006. Management of individual tree diameter growth and implications for pruning for Brazilian Eucalyptus grandisHill ex Maiden. Floresta, 36(3): 397-413.

PINAZO, M. et al.2008. Efecto de la intensidad de raleo sobre la compartimentalización y stock de carbono en plantaciones de Pinustaeda L. de 20 años en el norte de la provincia de Misiones. Revista de Investigaciones Agrarias, 36(1): 5-20.

REY, C. et al. 2001. Biomasa y acumulación de nutrientes en plantaciones de Pinus radiata D. Don del norte de España. Actas del III Congreso Forestal Español «Sierra Nevada 2001» Granada, 25-28 de Septiembre. Mesas 1 y 2. p. 500-504.

SIFIP. 2010. Sistema de información forestoindustrial provincial. Ministerio del Agro y la producción, provincia de Misiones, República Argentina. Consultado:http://extension.facfor. unam.edu.ar/sifip/inventario.

SCHNEIDER, P. et al.1991. Efeito da intensidade de desbaste sobre o crescimento e produção de povoamentos de Pinus elliottiiE. In: Simpósio de manejo de floresta plantadas, Santa María. Anais: UFSM. p.150-167.

SOIL SURVEY STAFF, 2006. Keys to soil taxonomy, $10^{\text {th }}$ ed. USDA-Natural Resources Conservation Service, Washington, DC.

SWORD, M.; GOELZ, J.; CHAMBERS, J. 2004. Long-term trends in loblolly pine productivity and stand characteristics in response to thinning and fertilization in the West Gulf region. Forest Ecology and Management, 192: 1 71-96.

TODARO, L.; MACCHIONI, N. 2011. Wood properties of young Douglas-fir in Southern Italy: results over a 12-year post-thinning period. European Journal of Forest Resource, 130:251261.

WODZICKI, T. 2001. Natural factors affecting wood structure. Wood Science Technology, 3:5-26. 